\title{
Plasma lipid concentrations in children with cystic fibrosis: the value of a high-fat diet and pancreatic supplementation
}

\author{
BY GRAHAM C. BURDGE ${ }^{1}$, ALISSA J. GOODALE ${ }^{1}$ CATHERINE M. HILL ${ }^{1}$, \\ PENNY J. HALFORD ${ }^{2}$, ELIZABETH J. LAMBERT ${ }^{1}$, ANTHONY D. POSTLE \\ AND CHRISTOPHER J. ROLLES \\ Departments of ${ }^{1}$ Child Health, Southampton General Hospital, Tremona Road, \\ Southampton SO9 $4 X Y$ and ${ }^{2}$ Human Nutrition, University of Southampton, Bassett Crescent East, \\ Southampton SO9 3TU
}

(Received 8 October 1992 - Revised 20 May 1993-Accepted 18 September 1993)

\begin{abstract}
Impaired digestion of dietary fat is an almost universal feature of cystic fibrosis (CF) which results in low concentrations of essential fatty acids in plasma lipids. We have evaluated the effect of a high-lipid diet and pancreatic enzyme supplementation, using enteric-coated microsphere preparations, on plasma lipid concentrations in paediatric CF patients. Absorption of dietary lipid was comparable between control and CF subjects. This resulted in plasma cholesterol, triacylglycerol, total phosphatidylcholine and individual phosphatidylcholine molecular species concentrations in CF patients which were in the same range as those in controls. Normal values for these variables were also found in patients with clinically detectable liver disease. These results show that present dietary management of CF patients supports normal plasma lipid concentrations.
\end{abstract}

Cystic fibrosis: Plasma lipids: Plasma phospholipids: Childhood

Impaired digestion of dietary fat as a consequence of pancreatic exocrine insufficiency is an almost universal feature of the disease process in cystic fibrosis (CF), resulting in decreased intake of energy, dietary essential fatty acids (EFA) and fat-soluble vitamins (Underwood et al. 1972; Farrell et al. 1985; Scott-Jupp et al. 1991). Reduced availability of EFA has been reported to alter patterns of circulating and tissue esterified fatty acids and may have profound implications in terms of membrane fluidity and cell signalling mechanisms (Exton, 1990). Alterations in these processes may be particularly important for inflammatory cells responding to chronic lung infections that are frequently found in $\mathrm{CF}$ patients.

Reduced concentrations of plasma EFA due to impaired uptake of dietary lipid may reflect the presence of liver disease. This is characterized by hepatic cirrhosis with focal lipid infiltration during the early stages. This occurs in up to $10 \%$ of CF patients, with increased incidence in the 16+ age group (Stern et al. 1976; Nagel et al. 1989; Scott-Jupp et al. 1991). The presence of fat infiltration suggests abnormal lipid metabolism. However, the role of liver disease in $\mathrm{CF}$ in altering patterns of plasma lipids has not been assessed.

Most attention has focused on linoleate $(18: 2 n-6)$, the major dietary $n-6$ EFA and precursor of arachidonate $(20: 4 n-6)$. Initial reports showed a decrease in plasma esterified $18: 2 n-6$ from $35 \%$ in control children to about $20 \%$ of total esterified fatty acids in CF patients, which affected all lipid classes (Kuo et al. 1962; Hubbard et al. 1977). More recent reports restricting this deficiency of $18: 2 n-6$ to cholesterol ester and phospholipid fractions (Rosenlund et al. 1974; Farrell et al. 1985) may reflect improvements in dietary management of CF patients, although details of diets used have rarely been reported.

Relatively recent improvements in the dietary management of children with $\mathrm{CF}$ include 
the introduction of both enteric-coated microsphere pancreatic enzyme preparations, and a diet high in lipid and energy. The effects of these advances on dietary lipid absorption and plasma lipid composition have not yet been systematically determined. Consequently, we have evaluated the efficiency of this dietary regimen upon the absorption of dietary lipid and the composition of plasma lipids in paediatric CF patients. In addition, we have resolved individual molecular species of plasma phosphatidylcholine (PC) in order to determine the EFA status of these patients and any possible effect of CF upon liver PC metabolism. These variables have also been evaluated in CF patients with clinically evident biliary cirrhosis. Our results show that present dietary management results in sufficient digestion and absorption of lipid to support normal plasma lipid concentrations in $\mathrm{CF}$ patients both with and without clinical liver disease.

\section{MATERIALS AND METHODS}

\section{Materials}

Methanol and trifluoroethanol were from Rathburn Ltd (Walkerburn, Peebleshire). Analar' grade chloroform and choline chloride were purchased from BDH Ltd (Poole, Dorset). All other chemicals, with noted exceptions, were obtained from Sigma (Poole, Dorset).

\section{Cystic fibrosis patients and control subjects}

Twenty-six cystic fibrosis patients aged between 3 and 20 years were recruited from the outpatient department at Southampton General Hospital, Southampton, Hants. This group included three patients (aged 15 and 16 years) who had hepatic cirrhosis. All these CF patients showed fat malabsorption at initial presentation, indicated by failure to thrive and symptomatic steatorrhoea. Blood samples from CF patients were obtained when they attended a routine clinic, held in the afternoon. Consequently, they were not asked to fast for $12 \mathrm{~h}$ before obtaining blood. Instead, they were instructed to eat a low-fat breakfast, containing $1167 \mathrm{~kJ}$ and $6 \mathrm{~g}$ total fat, and then fasted for a minimum of $4 \mathrm{~h}$ before blood was taken. Control subjects (aged 3-23 years; $n$ 15) were patients receiving elective surgery for conditions which did not involve changes in hepatic function. These subjects fasted for $12 \mathrm{~h}$ before blood was taken.

A subgroup ( $n$ 10) of the CF patients in whom circulating lipid concentrations were measured was assessed for their ability to absorb dietary fat. Fat absorption was also studied in an age-matched control group ( $n$ 10), which was not the same as that used for circulating lipid analysis.

Ethical approval for this study was granted by the joint ethical subcommittee of the Southampton and South West Hampshire District Health Authority and the University of Southampton.

\section{Dietary maintenance of $C F$ patients}

The general approach to the nutritional management of CF patients was a high-fat, highenergy diet designed to achieve $120-150 \%$ of the recommended daily allowance. All patients received pancreatic lipase, amylase and protease supplements in enteric-coated microsphere formulations (Duphar, Southampton, Hants; E. Merck, Four Marks, Alton, Hants; Ortho-Cilag, High Wycombe, Bucks.).

\section{Analysis of apparent lipid absorption by $C F$ patients}

Apparent lipid absorption was estimated from the difference between the dietary input of fatty acids and the loss of fatty acids in stool. Each subject completed a $7 \mathrm{~d}$ weighed food intake at home using digital scales under the supervision of a dietitian. Fat intake was 
calculated by computerized dietary analysis (Microdiet; Salford University). During the final $3 \mathrm{~d}$ of the period when food intake was weighed, stools were collected between carmine markers and used to measure faecal fat. It was assumed that all dietary fat not recovered in the stool was absorbed by the patient. This possibly resulted in an uncorrected overestimation of total dietary lipid absorption, for instance due to bacterial oxidation of fatty acids in the gut. However, since both CF and control groups were assessed in the same way, comparisons between subjects were valid. Briefly, $10 \mathrm{~g}$ homogenized faeces from $\mathrm{CF}$ patients and $20 \mathrm{~g}$ homogenized faeces from control subjects were saponified with $\mathrm{KOH}$ in methanol $(50 \mathrm{~g} / \mathrm{l})$ and then acidified with $4 \mathrm{M}-\mathrm{HCl}$. Fatty acids were then extracted with light petroleum (b.p. $40-60^{\circ}$ ) and their concentration determined by titration with $10 \mathrm{M}$-alcoholic $\mathrm{NaOH}$. Total fatty acid mass was calculated using the molecular weight of stearic acid.

\section{Plasma collection and lipid analysis}

Blood samples $(2 \mathrm{ml})$ were obtained by venepuncture. Clotting was prevented with EDTA and the plasma fraction collected by centrifugation at $2000 \mathrm{~g}$ for $20 \mathrm{~min}$ at $4^{\circ}$. Plasma samples were either mixed with sodium azide (final concentration $0.5 \mathrm{~g} / 1$ and stored at $4^{\circ}$ for analysis of total cholesterol, triacylglycerol (TAG) and PC concentrations or stored at $-20^{\circ}$ for $\mathrm{PC}$ molecular species analysis.

Total plasma cholesterol and TAG concentrations were determined using an RA1000 biochemical autoanalyser (Technicon, Basingstoke, Hants) using routine enzymic protocols. Total PC concentration was measured by colorimetric assay. Plasma samples ( $5 \mu \mathrm{l}$ ) containing $0.5 \mu \mathrm{l}$ Triton $\mathrm{X}-100$ were added to a ninety-six-well tissue culture plate (ICN Biomedicals, High Wycombe, Bucks.) and made up to $60 \mu \mathrm{l}$ with $100 \mathrm{~mm}$-Tris $-\mathrm{HCl}$, pH 7.0 containing $20 \mathrm{~mm}^{-\mathrm{CaCl}_{2}}$. Phospholipase D (EC 3.1.4.4; from Streptomyces chromofusaes $\left(2 \times 10^{-3} \mathrm{U} /\right.$ well; Genzyme, West Malling, Kent) was added and the reaction mixture incubated at $42^{\circ}$ for $50 \mathrm{~min}$. Chromogen $(100 \mu 1 ; 100 \mathrm{~mm}-\mathrm{Tris}-\mathrm{HCl}$, pH 8.0 , containing $0.2 \mathrm{~g}$ phenol $/ 1,1.5 \mathrm{~mm}$-4-aminoantipyrene, $0.2 \mathrm{U} / \mathrm{ml}$ choline oxidase (EC 1.1.3.17; Boehringer Mannheim, Lewes, East Sussex) and 18.4 U horseradish peroxidase $E C 1.11 .1 .7$ ) was added to each well and the reaction continued at $37^{\circ}$ for a further $50 \mathrm{~min}$. PC concentration was determined by absorbance at $492 \mathrm{~nm}$ against a standard curve of $0-50$ nmol dipalmitoyl PC (PC16:0/16:0).

\section{HPLC analysis of the PC molecular species composition of plasma}

Plasma samples $(200 \mu \mathrm{l})$ in $1.0 \mathrm{ml}$ saline $(9 \mathrm{~g} \mathrm{NaCl} / 1)$ were extracted with chloroformmethanol (Bligh \& Dyer, 1959). Dimyristoyl PC (PC14:0/14:0; $50 \mathrm{nmol}$ ) was added as internal standard. PC was isolated from the chloroform phase on a $100 \mathrm{mg}$ Varian BondElut $\mathrm{NH}_{2}$ disposable cartridge (Jones Chromatography Ltd, Hengoed, Mid Glamorgan; Caesar et al. 1988). The PC fraction was collected, dried under $\mathrm{N}_{2}$ at $40^{\circ}$ and dissolved in $40 \mu \mathrm{l}$ trifluoroethanol. PC molecular species were resolved on a $250 \mathrm{~mm} \times$ $4.6 \mathrm{~mm}$ i.d. Apex II ODS column (Jones Chromatography) at $50^{\circ}$ using a mobile phase of methanol-water $(92 \cdot 5: 7 \cdot 5, \mathrm{v} / \mathrm{v})$ containing $40 \mathrm{~mm}$-choline chloride, at a flow rate of $1 \mathrm{ml} / \mathrm{min}$ (Postle, 1987). PC molecular species were quantified by post-column fluorescence derivatization with 1,6-diphenyl-1,3,5-hexatriene (Postle, 1987).

\section{Statistical methods}

Results of analysis of plasma lipid samples from $\mathrm{CF}$ and control subjects were divided by age into prepubertal (3-10 years) and adolescent/adult ( $>10$ years) subgroups to eliminate any influence of sexual maturation upon circulating lipids when comparing subject groups. Data which were normally distributed were analysed using the unpaired Student's $t$ test. 
Data which were not normally distributed were analysed by the Mann-Whitney U test. $P<0.05$ was regarded as significant.

\section{RESULTS AND DISCUSSION}

Inefficient digestion of dietary fat due to pancreatic insufficiency results in reduced uptake of lipid by the gut. The majority of previous studies on circulating lipid concentrations in $\mathrm{CF}$ were conducted before the implementation of the present dietary regimen of a high-fat, high-energy diet and development of efficient pancreatic enzyme supplements. The total lipid intake (analysed by the Mann-Whitney U Test) was similar in both the CF group $(73.6(\mathrm{SD} 31.6) \mathrm{g} / \mathrm{d})$ and controls $(65.1(\mathrm{SD} 24.9) \mathrm{g} / \mathrm{d})$. The apparent absolute fat absorption by CF patients ( 55.3 (SD 23.5$) \mathrm{g} / \mathrm{d}$ ) was slightly lower than by control subjects (61.7 (SD 24.0) $\mathrm{g} / \mathrm{d})$, although these values were not significantly different. The relative absorption of fat (78.1 (SD 18.3, range $40.8-96.0) \%$ ) by the CF group, however, was significantly lower compared with control subjects $(94 \cdot 7$ (SD 3.2, range 89.6-99.7)\%). These results indicate that $\mathrm{CF}$ patients continued to malabsorb fat, relative to dietary intake, despite maintenance with pancreatic enzyme supplementation and a high-energy diet. However, this treatment regimen permitted sufficient dietary fat to be digested for a nearly normal daily uptake of fat to be achieved in the CF group.

Total plasma cholesterol, TAG and PC concentrations of CF patients were within the range of values obtained from control subjects (Table 1). Previous studies have reported decreased plasma cholesterol concentrations in CF, especially in older patients (Vaughan et al. 1978). Our results showed a slightly lower plasma cholesterol concentration in the adolescent $\mathrm{CF}$ age group which was not sufficient to achieve statistical significance (Table 1). The concentration of TAG was consistently slightly elevated in the CF group compared with controls (Table 1), although still within the normal range; this was probably attributable to the CF patients not having been fasted as long as the control group before obtaining blood. Therefore, a high-lipid, high-energy diet combined with pancreatic enzyme supplementation was sufficient to support normal plasma PC, cholesterol and TAG concentrations.

Previous studies have shown significantly decreased plasma cholesterol ester, TAG and PC $n$-6 EFA concentrations, especially 18:2n-6, in CF patients (Kuo et al. 1962; Rosenlund et al. 1974; Hubbard et al. 1977; Farrell et al. 1985). We have measured the concentration of intact individual molecular species of plasma PC in order to assess the uptake from the diet and supply to the tissues of EFA. Eight PC molecular species were consistently identified (Table 2) which accounted for more than $95 \%$ of total plasma PC and consisted mainly of $18: 2 n-6,18: 1 n-9$ and $20: 4 n-6$ PC species. The concentration of individual PC molecular species did not differ significantly between control and CF subjects (Table 2), indicating that normal circulating concentrations of $n-3$ and $n-6$ EFA can be achieved in CF patients by present dietary management. Elevated plasma concentrations of $20: 3 n-9$, the chain elongation product of $18: 1 n-9$, are recognized as a marker for EFA deficiency in many studies. Molecular species containing 20:3n-9 were consistently identified in the present study, but never comprised more than $0 \cdot 3 \%$ of total plasma PC. Moreover, values for 20:3n-9-containing PC species were identical between $\mathrm{CF}$ and control subjects. Consequently, these results did not suggest any clinically significant EFA deficiency in these CF patients. These results are consistent with the results of previous studies in which $20: 3 n-9$ was only detected in low concentrations in plasma from CF patients (Hubbard et al. 1977; Farrell et al. 1985). One potentially beneficial effect of achieving normal plasma concentrations of EFA, especially $18: 2 n-6$, is the maintenance of appropriate membrane phospholipid compositions in inflammatory cells. Kang et al. (1992) have recently 
Table 1. Concentrations (mmol/l) of cholesterol, triacylglycerol (TAG), and phosphatidylcholine $(P C)$ in plasma from control subjects and subjects with cystic fibrosis*

(Mean values and standard deviations)

\begin{tabular}{|c|c|c|c|c|c|c|c|c|}
\hline \multirow{3}{*}{$\begin{array}{l}\text { Subject age (years) ... } \\
n \ldots\end{array}$} & \multicolumn{4}{|c|}{ Control } & \multicolumn{4}{|c|}{ Cystic fibrosis } \\
\hline & \multicolumn{2}{|c|}{$\begin{array}{c}3-10 \\
7\end{array}$} & \multicolumn{2}{|c|}{$\begin{array}{c}>10 \\
8\end{array}$} & \multicolumn{2}{|c|}{$\begin{array}{c}3-10 \\
14\end{array}$} & \multicolumn{2}{|c|}{$\begin{array}{c}>10 \\
12\end{array}$} \\
\hline & Mean & SD & Mean & $\mathrm{SD}$ & Mean & $\mathrm{SD}$ & Mean & SD \\
\hline Cholesterol & $3 \cdot 6$ & 0.6 & 3.6 & 0.8 & 2.9 & 0.6 & $2 \cdot 8$ & 0.7 \\
\hline TAG & 0.5 & $0 \cdot 1$ & 0.6 & 0.4 & 0.7 & 0.3 & 0.6 & 0.3 \\
\hline $\mathrm{PC}$ & 1.6 & 0.26 & 1.8 & 0.3 & 1.7 & $0 \cdot 4$ & $1 \cdot 6$ & 0.4 \\
\hline
\end{tabular}

* The concentrations of lipids in three cystic fibrosis patients with clinically detectable liver disease were: cholesterol 2.6, 2.5 and $2.8 \mathrm{mmol} / 1$; TAG $0.2,0.8$ and $0.4 \mathrm{mmol} / \mathrm{l}$; PC $1.4,1.6$ and $1.8 \mathrm{mmol} / 1$.

Table 2. Phosphatidylcholine (PC) molecular species composition ( $\mathrm{nmol} / \mathrm{ml}$ ) of plasma from controls and cystic fibrosis $(C F)$ patients

(Values are means and standard deviations with the exception of the column showing the analysis of plasma PC molecular species from CF patients with clinically detectable liver disease where individual values are presented)

\begin{tabular}{|c|c|c|c|c|c|c|c|c|c|}
\hline \multirow{3}{*}{$\begin{array}{l}\text { Subject age (years) ... } \\
n \ldots \\
\text { Molecular species* }\end{array}$} & \multicolumn{4}{|c|}{ Control } & \multicolumn{5}{|c|}{ Cystic fibrosis } \\
\hline & \multicolumn{2}{|c|}{$\begin{array}{c}3-10 \\
6\end{array}$} & \multicolumn{2}{|c|}{$\begin{array}{c}>10 \\
6\end{array}$} & \multicolumn{2}{|c|}{$\begin{array}{c}3-10 \\
11\end{array}$} & \multicolumn{2}{|c|}{$\begin{array}{c}>10 \\
11\end{array}$} & \multirow{2}{*}{$\begin{array}{l}\text { Patients with } \\
\text { clinical liver } \\
\text { disease }(n 3)\end{array}$} \\
\hline & Mean & SD & Mean & SD & Mean & SD & Mean & $\mathrm{SD}$ & \\
\hline $16: 0 / 22: 6$ & 89 & 39 & 112 & 42 & 89 & 53 & 108 & 40 & $84,54,89$ \\
\hline $16: 0 / 20: 4$ & 202 & 80 & 232 & 132 & 109 & 56 & 245 & 84 & $199,121,309$ \\
\hline $16: 0 / 18: 2$ & 649 & 110 & 926 & 386 & 709 & 209 & 828 & 196 & $588,659,1001$ \\
\hline $16: 0 / 16: 0$ & 38 & 17 & 37 & 16 & 55 & 14 & 46 & 28 & $27,40,63$ \\
\hline $16: 0 / 18: 1$ & 329 & 76 & 422 & 205 & 419 & 129 & 515 & 191 & $494,461,552$ \\
\hline $18: 0 / 22: 6$ & 38 & 17 & 58 & 16 & 46 & 27 & 58 & 20 & $49,72,59$ \\
\hline $18: 0 / 20: 4$ & 116 & 47 & 141 & 94 & 95 & 70 & 127 & 59 & $90,63,180$ \\
\hline $18: 0 / 18: 2$ & 324 & 72 & 484 & 172 & 426 & 152 & 460 & 128 & $331,306,483$ \\
\hline
\end{tabular}

* Molecular species of phosphatidylcholine are designated by the fatty acids on their sn-1 and sn-2 acyl positions.

suggested that reduced tissue 18:2n-6 concentration was due to a specific effect of CF. However, this seems unlikely since the low plasma 18:2n-6 concentrations previously reported (Kuo et al. 1962; Rosenlund et al. 1974; Hubbard et al. 1977; Farrell et al. 1985) were prevented by improved dietary management alone.

The CF group in the present study included three patients with hepatic cirrhosis and lipid infiltration who showed abnormal serum liver function tests, ultrasound scan and clinical assessment. The concentrations of cholesterol, TAG, total PC and individual PC molecular species in plasma from these patients were within the ranges of $\mathrm{CF}$ patients with no liver disease and the control subjects (Tables 1 and 2). Present dietary management of CF patients, therefore, provides sufficient dietary fat to support apparently normal plasma lipid concentrations, even in cases of severe hepatic cirrhosis. This suggests that the primary 
cause of abnormal plasma lipid patterns in CF reported previously was probably poor utilization of dietary lipid and not a specific effect of CF on hepatic lipoprotein synthesis and secretion. Furthermore, since the composition of plasma PC molecular species was essentially unaffected it seems unlikely that $\mathrm{CF}$ affects $\mathrm{PC}$ synthesis directly. An alternative explanation is that, due to the focal nature of hepatic lipid infiltration in $\mathrm{CF}$, sufficient unaffected tissue remained in these patients to support normal liver lipid metabolism. Since normal circulating lipid concentrations were maintained in CF patients with hepatic lipid accumulation, it is unlikely that build-up of lipid in liver tissue is caused by impaired lipoprotein secretion, but may possibly be due to abnormal fat catabolism.

We would like to thank the staff of the Department of Chemical Pathology for their assistance in measuring plasma triacylglycerols and cholesterol.

\section{REFERENCES}

Bligh, E. G. \& Dyer, W. S. (1959). A rapid method of total lipid extraction and purification. Canadian Journal of Biochemistry 37, 911-923.

Caesar, P. A., Wilson, S. J., Normand, I. C. S. \& Postle, A. D. (1988). A comparison of the specificity of phosphatidylcholine synthesis by human fetal lung maintained in either organ or organotypic culture. Biochemical Journal 253, 451-457.

Exton, J. H. (1990). Signalling through phosphatidylcholine breakdown. Journal of Biological Chemistry $265,1-4$.

Farrell, P. M., Mischler, E. H., Engle, M. J., Brown, D. J. \& Lau, S.-M. (1985). Fatty acid abnormalities in cystic fibrosis. Pediatric Research 19, $104-109$.

Hubbard, V. S., Dunn, G. D. \& Di Sant' Agnese, P. A. (1977). Abnormal fatty acid composition of plasma lipids in cystic fibrosis. Lancet ii, 1302-1304.

Kang, J. X., Man, S. F. P., Brown, N. E., Labrecque, P. A. \& Clandinin, M. T. (1992). The chloride channel blocker anthracene 9-carboxylate inhibits fatty acid incorporation into phospholipid in cultured human airway epithelial cells. Biochemical Journal 285, 725-729.

Kuo, P. T., Huang, N. N. \& Bassett, D. R. (1962). The fatty acid composition of the serum chylomicrons and adipose tissue of children with cystic fibrosis of the pancreas. Journal of Pediatrics 60, 394-403.

Nagel, R. A., Westaby, D., Javaid, A., Kavani, J., Meire, H. B., Lombard, M. G., Wise, A., Williams, R. \& Hodson, M. E. (1989). Liver disease and bile duct abnormalities in adults with cystic fibrosis. Lancet ii, 1422-1424.

Postle, A. D. (1987). Method for the analysis of individual molecular species of phosphatidylcholine by highperformance liquid chromatography with post-column fluorescence detection. Journal of Chromatography $\mathbf{4 1 5}$, 41-51.

Rosenlund, M. L., Kim, H. K. \& Kritchevsky, D. (1974). Essential fatty acids in cystic fibrosis. Nature 251, 719.

Scott-Jupp, R., Lama, M. \& Tanner, M. S. (1991). Prevalence of liver disease in cystic fibrosis. Archives of Disease in Childhood 66, 698-701.

Stern, R. C., Stevens, D. P., Boat, T. F., Doershuk, C. F., Izant, R. J. \& Matthews, L. D. (1976). Symptomatic hepatic disease in cystic fibrosis: incidence, course and outcome of portal systemic shunting. Gastroenterology 70, 645-649.

Underwood, B. A., Denning, C. R. \& Navab, M. (1972). Polyunsaturated fatty acids and tocopherol levels in patients with cystic fibrosis. Annals of the New York Academy of Sciences 203, 237-247.

Vaughan, W. J., Lindgren, F. T., Whalen, J. B. \& Abraham, S. (1978). Serum lipoprotein concentrations in cystic fibrosis. Science $199,783-785$. 